\title{
The head turn paradigm to assess auditory laterality in cats: influence of ear position and repeated sound presentation
}

\author{
Wiebke S Konerding ${ }^{1}{ }^{,}$Elke Zimmermann ${ }^{2}$, Eva Bleich $^{3}{ }^{4}$ Hans Hedrich ${ }^{3}$, Marina Scheumann ${ }^{\text {Corresp. }}{ }^{2}$ \\ 1 Institute of AudioNeuro Technology and Department of Experimental Otology, Hannover Medical School, Hannover, Germany \\ 2 Institute of Zoology, University of Veterinary Medicine Hannover, Hannover, Germany \\ 3 Institute for Laboratory Animal Science and Central Animal Facility, Hannover Medical School, Hannover, Germany \\ Corresponding Author: Marina Scheumann \\ Email address: marina.scheumann@tiho-hannover.de
}

In most humans, speech is predominantly processed by the left hemisphere. This auditory laterality was formerly thought to be an exclusive human characteristic, but is now suggested to have pre-human origins. In studies on auditory laterality in nonhuman animals, the head turn paradigm has become very popular due to its non-invasive character. Although there are implications that the head turn direction indicates functional dominance of the contralateral hemisphere in processing a given sound, the validity of the paradigm is under debate. To validate the paradigm via comparison with imaging or electrophysiological methods, it is first necessary to establish turning biases at the individual level. Recently, the domestic cat, a common model in hearing research, has been found to show turning biases at the group level. To assess individual turning asymmetries in cats, we repeatedly presented kitten isolation calls and assessed whether differences in conveyed arousal changed the previously described left-wards lateralisation of conspecific vocalizations. Based on responses to 50 playback presentations ( 25 of high and 25 of low arousal), we calculated individual head turn indices. Based on the total data set, we found no consistent individual turning bias, irrespective of call category or sex of the receiver. Although the playback paradigm was chosen carefully to reduce any effects of lateralized loudness perception or changes in motivation due to habituation, individual head turn biases changed significantly in concordance with habituation to repeated playback-presentations and was predictable by small deflections in ear position prior to listening. When splitting the data set according to a decline in responsiveness after 7 playback presentations, we revealed an initial left turning bias for most of our subjects (i.e. significant at the group level). We propose that this left turning bias is related to right hemisphere dominance in processes like vigilance behaviour or general arousal rather than on auditory processing, as such. Our findings suggest that both the experimental sequence and sound level differences, induced by asymmetric ear positions, strongly 
influence the outcome of the head turn paradigm and should be taken into account when evaluating auditory laterality at the behavioural level. 
1 The head turn paradigm to assess auditory laterality in cats: influence of

4 Wiebke S Konerding ${ }^{1}$, Elke Zimmermann ${ }^{2}$, Eva Bleich ${ }^{3}$, Hans-Jürgen Hedrich ${ }^{3}$, Marina 5 Scheumann $^{2 *}$

$7{ }^{1}$ Institute of AudioNeuroTechnology and Department of Experimental Otology, Hannover

8 Medical School, Hannover, Germany

9 2Institute of Zoology, University of Veterinary Medicine Hannover, Hannover, Germany

$10{ }^{3}$ Institute for Laboratory Animal Science and Central Animal Facility, Hannover Medical

11 School, Hannover, Germany

12

$13{ }^{*}$ Corresponding author

14 Marina Scheumann

15 E-mail: marina.scheumann@tiho-hannover.de

16

17

18

19

20

21 


\section{Abstract}

In most humans, speech is predominantly processed by the left hemisphere. This auditory

24 laterality was formerly thought to be an exclusive human characteristic, but is now suggested to

25 have pre-human origins. In studies on auditory laterality in nonhuman animals, the head turn

26 paradigm has become very popular due to its non-invasive character. Although there are

27 implications that the head turn direction indicates functional dominance of the contralateral

28 hemisphere in processing a given sound, the validity of the paradigm is under debate. To validate

29 the paradigm via comparison with imaging or electrophysiological methods, it is first necessary

30 to establish turning biases at the individual-level. Recently, the domestic cat, a common model in

31 hearing research, has been found to show turning biases at the group-level. To assess individual

32 turning asymmetries in cats, we repeatedly presented kitten isolation calls and assessed whether

33 differences in conveyed arousal changed the previously described left-wards lateralisation of

34 conspecific vocalizations. We calculated individual head turn indices to 50 playback

35 presentations (25 of high and 25 of low arousal). Based on the total data set, we found no

36 consistent individual turning bias, irrespective of call category or sex of the receiver. Although

37 the playback paradigm was chosen carefully to reduce any effects of lateralized loudness

38 perception or changes in motivation due to habituation, individual head turn biases changed

39 significantly in concordance with habituation to repeated playback-presentations and was

40 predictable by small deflections in ear position prior to listening. When splitting the data set

41 according to a decline in responsiveness after 7 playback presentations, we revealed an initial left

42 turning bias for most of our subjects (i.e. significant at the group-level). We propose that this left

43 turning bias is related to right hemispheric dominance in processes like vigilance behaviour or 
44 general arousal rather than on auditory processing, as such. Our findings suggest that both the

45 experimental sequence and sound level differences, induced by asymmetric ear positions, 46 strongly influence the outcome of the head turn paradigm and should be taken into account when 47 evaluating auditory laterality at the behavioural level.

\section{Introduction}

A characteristic feature of human language is the auditory laterality (i.e. left hemispheric

51 dominance) for speech processing which is supported by several behavioural and neurological 52 studies (Dehaene-Lambertz et al. 2002; Fischer et al. 2009; Hugdahl \& Westerhausen 2015). To 53 study preverbal laterality in human infants, Young and colleagues $(1983 ; 1990)$ introduced the 54 so-called head turn paradigm (or head-orienting-asymmetry paradigm). Thereby, a sound is played back from a loudspeaker $180^{\circ}$ behind the subject. The unconditioned head turn response to the acoustic stimulus is suggested to indicate the dominance of the contralateral hemisphere in processing the auditory stimulus. Young and colleagues showed that human infants turned their head to the right when listening to speech sounds, suggesting that this paradigm is suited to reveal a left hemispheric dominance for speech processing.

Due to its non-invasive character and its simplicity, the head turn paradigm has become 61 popular to study auditory laterality in non-human mammals ranging from mammals (Gil-da62 Costa \& Hauser 2006; Hauser \& Andersson 1994; Leliveld et al. 2010; Scheumann \& 63 Zimmermann 2008), to birds (Palleroni \& Hauser 2003), to amphibians (Xue et al. 2015). For 64 example in rhesus macaques (Hauser \& Andersson 1994) and sea lions (Böye et al. 2005) a right 65 ear preference for conspecific versus heterospecific calls was shown, suggesting a left 66 hemispheric dominance for processing conspecific vocalisations similar to human speech 
67 processing (for review see Ocklenburg et al. 2013; Taglialatela 2007). However, the outcomes

68 using the head turn paradigm are not consistent and the interpretation of the paradigm is further

69 complicated as the head turn asymmetries in response to species-specific calls are influenced by

70 a variety of different intrinsic and extrinsic factors. For example, specific acoustic properties of

71 the calls (Ghazanfar et al. 2001; Hauser et al. 1998; Ratcliffe \& Reby 2014; Siniscalchi et al.

72 2012), familiarity with the sender (Basile et al. 2009a; Basile et al. 2009b; Lemasson et al. 2010),

73 characteristics of the sender (Leliveld et al. 2010; Scheumann \& Zimmermann 2008) or

74 emotional valence (Basile et al. 2009b; Reinholz-Trojan et al. 2012; Xue et al. 2015) alter the

75 species-specific asymmetries in response to conspecific calls. Thus, it is argued that the

76 asymmetries revealed by the head turn paradigm might be related to the induced arousal, based

77 on the conveyed behavioural relevance of a given sound. This is partly consistent with findings

78 in humans, as speech laterality can be shifted away from the typical left hemispheric dominance

79 when listeners focus their attention on the emotional (i.e. prosodic) information of speech rather

80 than its linguistic content (Ley \& Bryden 1982). The head turn paradigm might therefore be

81 more suited to revealing lateralization due to induced arousal rather than being an indicator for

82 lateralization of species specific calls, comparable to human speech lateralization.

83 The validity of the paradigm has been challenged by a study in adult humans, which did

84 not show the expected right ear turning bias in a supermarket environment (Fischer et al. 2009).

85 The authors concluded that factors like attention and experimental situation significantly

86 influence the outcome of the head turn paradigm (Fischer et al. 2009). Thus, further

87 methodological assessments will be necessary to prove the validity of the paradigm. To validate

88 the paradigm it would be necessary to compare individual turning-biases to brain imaging or

89 electrophysiological data derived from the same individuals (Fischer et al. 2009). However, to 
90 the best of our knowledge, no study has presented statistical evidence for individual head turn

91 biases. The reason for this is that authors used only a low number of stimuli due to their concern

92 that animals would stop responding after only view repetitions (Hauser \& Andersson 1994;

93 Reinholz-Trojan et al. 2012; Teufel et al. 2007) which makes individual analysis impossible

94 (Leliveld et al. 2010; Lemasson et al. 2010; Siniscalchi et al. 2008). Lemasson et al. (2010)

95 provided a study where each sound was presented 40 times, which indicates that individual head

96 turn biases may be achieved. However, the authors did not report individual asymmetries and

97 also did not comment on potential habituation effects. Thus, the aim of our study was to derive

98 individual head turn biases towards species-specific calls of different arousal and to investigate

99 the effect of repeated sound presentations for the domestic cat The cat is a common model in

100 hearing research (e.g. Eggermont 1998; Heffner \& Heffner 1985; Land et al. 2016) and shows

101 lateralized motor behaviour (Konerding et al. 2012; Wells \& Millsopp 2009; Wells \& Millsopp

102 2012).

103 Recently, Siniscalchi and colleagues (2016) published that cats turn their head to the right

104 in response to conspecific calls, indicating the typical left hemispheric dominance in processing

105 species-specific calls. However, only purring and meow sounds elicit right ear preferences,

106 whereas growls showed a tendency in the opposite direction. The authors argued that this might

107 be explained by different emotional meaning (Siniscalchi et al. 2016). Growls might cause a shift

108 to the right hemisphere due to higher levels of induced arousal or behavioural relevance.

109 Nevertheless, the acoustic properties of these call types are very different (noisy to tonal calls),

110 which might also affect hemispheric processing. Thus, to address the impact of induced arousal

111 on auditory laterality in response to species-specific calls, it would be useful to investigate

112 auditory laterality in response to the same call type recorded in low versus high arousal states. 
113 In our previous publications, we already showed that kitten isolation calls show changes

114 in acoustic properties during low versus high arousal behavioural conditions (Scheumann et al.

115 2012) and that these differences in conveyed arousal induced concordant differences in

116 responsiveness in female cats (Konerding et al. 2016). Thereby, females oriented significantly

117 faster towards the sound-source to high versus low arousal kitten calls. To assess the impact of

118 induced arousal on the lateralization of conspecific calls, we investigated potential differences in

119 head turn direction toward these two call categories. that did only differ in a defined set of

120 acoustic parameters, related to the arousing property of the eliciting behavioural situation

121 (Scheumann et al. 2012) and have been found to influence the urgency to respond in female but

122 not male cats (Konerding et al. 2016). We expected a shift to a left ear preference (i.e. more left

123 than right head turns) for high compared to low arousal calls in female cats, only. As males

124 showed no behavioral differences between either arousal categories (Konerding et al. 2016) we

125 expect them to show the typical right ear/ left hemispheric dominance to both types of this 126 species-specific vocalization.

127 As we were the first to assess individual head turn responses, we decided to also look at

128 potential influencing factors in more detail. The first factor is the effect of overall habituation to

129 the playback-situation. This can be expected to have a greater impact on the results in an

130 individual based approach than when combining several animals, which are tested only once per

131 call type. The second is related to our animal model. As domestic cats have highly movable

132 pinnae that are sometimes switched back and forth within milliseconds, we were concerned with

133 potential loudness differences between the ears, based on ear position. As we took care to reduce

134 the risk of habituation and monitored the ear position to choose the best time-point for playback- 
135 presentation, we expected these factors to have of only minor impact on the result of our 136 experiments.

138 Material and methods

\section{Subjects}

We tested 15 adult cats ( 8 males, 7 females) aged 1 to 8 years $\left(\operatorname{mean}_{\mathrm{m}}=2.6, \operatorname{mean}_{\mathrm{f}}=3.3\right)$.

141 Housing conditions were the same, as described in (Konerding et al. 2016). All subjects were not

142 neutered/ castrated and originated from and were kept at the breeding facility of the Central

143 Animal Facility of the Hannover Medical School. Adult cats lived in same-sex groups of 2-5

144 individuals, with changing composition based on breeding schedules. The cats were kept indoors

145 in a controlled environment (light-dark cycle $12: 12,22 \pm 2{ }^{\circ} \mathrm{C}, 55 \pm 10 \%$ humidity). The rooms

146 (12.5 $\mathrm{m}^{2}-20.6 \mathrm{~m}^{2}$, height: $\left.2.6 \mathrm{~m}\right)$ were enriched with wooden boxes, tables and shelves, plastic

147 toys and bars for scratching. As an additional heat source, each room was equipped with an

148 infrared lamp. The cats were fed daily with tinned (Whiskas ${ }^{\circledR}$ tins, Mars GmbH, Verden,

149 Germany) and dry cat food (SDS Pet Food, Special Diets Services, Witham, Essex, UK) and

150 were provided with water ad libitum. The animal husbandry fulfilled all recommendations for

151 domestic cats as required by the guidelines of the European Union (ETS 123, Directive

152 2010/63/EU) and was approved by the local veterinary authority (No. 42500/1H).

153

\section{Preparation of playback stimuli}

The playback stimuli originated from a previous study on the acoustic communication of

156 the domestic cat (Konerding et al. 2016). They were recorded from kittens aged 9 to 11 days 
157 during separation conditions, varying only with regard to the amount of handling, either without

158 (low arousal) or with (high arousal) handling by a human observer (for recording details see

159 Scheumann et al. 2012). We used 14 calls from 7 senders as playback stimuli: One call of low

160 and one of high arousal from each sender (Fig 1). Multi-parametric sound analyses revealed that

161 specific acoustic cues of the kitten isolation call (i.e. call duration, voicing and fundamental

162 frequency) are distinct with regard to the arousal state of the sender (paired t-tests: $\mathrm{p} \leq 0.033$;

163 Fisher Omnibus test: $\chi 2=44.7, \mathrm{df}=20, \mathrm{p}=0.001$; Scheumann et al. 2012). The stimuli were played

164 back at a standardised sound pressure level (SPL) of $70 \pm 2 \mathrm{~dB}$ at a distance of $1.8 \mathrm{~m}$ (i.e. licking

165 distance, see below) from the loudspeaker (quadral Argentum 02.1, quadral GmbH \& Co. KG;

166 RMS fast measurement: Brüel and Kjær 2610, highpass filter: $22.4 \mathrm{~Hz}$ ), to match the loudness of

167 natural kitten vocalisations (Romand \& Ehret 1984).

168

169

\section{Playback experiments and experimental set-up}

A subset of the behavioural recordings has already been analysed to assess the impact of conveyed arousal on the latency to orient towards the loudspeaker (Konerding et al. 2016). Thus,

172 the methods have already been described in detail. In short, the cats were tested in a sound

173 attenuated surrounding. The experimental cage was equipped with a drinking bottle containing a

$174 \mathrm{milk} /$ water mixture and at the opposite side the loudspeaker was placed behind an opening in the

175 foam of one movable wall. Thereby, sounds were perceived from the defined direction $\left(180^{\circ}\right.$ 176 behind) and distance $(1.8 \mathrm{~m})$ while the cat was within licking distance. Before the actual

177 experiments started, each animal got accustomed to the experimental set-up.

178 To reduce the risk of habituation, we limited the number of stimuli presented per trial and 179 the number of trials per week. Each cat was tested individually 2 to 4 times a week and usually 
$180(61 \%)$ only one stimulus (maximum 4) was played during each trial. When more stimuli were

181 played, the inter-stimulus interval was at least 1 minute. The stimuli were played back in pseudo-

182 randomised order. The stimulus was initiated by the experimenter based on videographic

183 monitoring of the animal, i.e. when the subject was within licking distance of the drinking bottle,

184 with its body aligned closely to the bottle-loudspeaker axis and its head held approximately

185 straight (cage bars served as visual reference). The freedom for head movements was restricted

186 by additional wire mesh to an angle of +-30 degree (Fig. 2). As the cat has highly movable

187 pinnae (Populin \& Yin 1998), we took care that not only the head was straight but also the ears

188 had the same distance to the loudspeaker when presenting a given call, i.e. the tips of the pinnae

189 were parallel to the bars of the test cage. Still, we could not avoid small deflections (see video

190 analysis) in the ear position during listening and included this as a potential confounding factor

191 in the statistical analysis. The experiments of one subject were completed when 25 stimulus

192 presentations of each category (i.e. 25 low and 25 high arousal calls) had been scored in the 193 video analysis.

195 Video analysis

196 The video analyses were performed blind to the respective playback stimulus (i.e. without

197 acoustic information). Thus, all experiments were scanned for playback presentations indicated

198 by the lightening of a diode (invisible to the subject). We analysed only those presentations

199 where a subject was in drinking distance of the bottle at the first flashing of the diode. We scored

200 the first head turn response within 5 seconds of stimulus onset (Interact 32 software, Version 8,

201 Mangold, Arnstorf, Germany;e.g. (Basile et al. 2009a; Siniscalchi et al. 2012; Siniscalchi et al.

202 2008). The ear position prior to listening to the calls was assessed based on any deflections from 
203 a parallel position, using the cage bars as reference (see Fig. 2). We noted whether the left or the 204 right pinna was directed more closely to the loudspeaker at the first flashing of the diode (i.e. 205 defined as prior to listening).

206 To test for inter-observer reliability, $25 \%$ of the playback presentations (i.e. complete 207 data of 2 males and 2 females) were reanalysed by a second observer. The comparison of the 208 indices (see Statistical analysis) for both the head turn direction and the ear position derived by 209 the two observers correlated significantly, indicating a high inter-observer reliability (two-tailed

210 Pearson correlation, linear regression: head turn: $\mathrm{p}=0.02, \mathrm{r}=0.98, \mathrm{y}=0.02+0.83 \mathrm{x}$; ear

211 position: $\mathrm{p}<0.01, \mathrm{r}=0.10, \mathrm{y}=-0.01+1.00 \mathrm{x})$.

212

\section{Statistical analysis}

We calculated for each individual separately (for raw data see supplement table S1), the 215 head turn index (HTI) from the numbers of left $(\mathrm{L})$ and right $(\mathrm{R})$ head turns as HTI $=(\mathrm{R}-\mathrm{L})$ / $(\mathrm{R}+\mathrm{L})$. The HTI ranges from -1 , for exclusive left bias, to +1 , for exclusive right bias, with 0 indicating equal distribution of left and right turns. Individual turning biases were assessed via an exact binomial test. Accordingly, we defined individuals with a significant bias as left (negative HTI) lateralized, right (positive HTI) lateralised, or non-lateralised $(p>0.05)$. HTIs were assessed for the two call types and the two sexes separately. Asymmetries at group-level were assessed via two-tailed, one-sample t-tests, to report significant deviations from zero. To investigate potential confounding factors, we performed a binomial GLMM (Zuur et al. 2009) to assess the influence of the explanatory variables: Sex (male/female), Arousal (high/low), Order

224 (playback presentation \#1-50) and Initial Ear Position (straight, right, left) on the test variable 225 Head Turn Direction (right, left) and used Subject as random variable. 
effect of repeated sound presentation on the number of responding individuals and the HTI

228 (GraphPad Prism 5). By splitting the data set when only about $50 \%$ of the subjects were

229 responding to the playback presentation (see Results), we assessed individual HTIs for both the

230 first 7 and the following 7 presentations. Based on these HTIs, we assessed whether potential

231 group-level biases in head turn responses changed based on habituation (paired t-test). Biases at

232 individual-level could not be statistically assessed after splitting the data set, due to the

233 remaining small sample size. For comparison with other head turn studies, we also assessed

234 group-level HTIs, based on the first head turn response of each individual to each of the two call

235 categories.

236 To investigate the potential influence of ear position prior to listening to the playback

237 calls we analysed whether the prevalence of same-side turns was significantly higher than 238 expected by chance (t-test vs $50 \%$ ) and separately computed HTIs only for those occasions 239 where the ear position was straight.

Prior to each analysis, we checked normality using the Kolmogorov-Smirnov test

241 (normality was assumed if $\mathrm{p}>0.05$ ) and used non-parametric versions of the test statistic,

242 whenever applicable. The level of significance was set at $\mathrm{p}<0.05$ and whenever available, we 243 performed exact versions of the test statistics.

\section{Results}

\section{Head turn bias}

247 On individual-level, two cats (one male, one female) showed a significant head turn bias 248 in response to low arousal calls, only (female: HTI $=1.00, \mathrm{n}=7$ head turns; male: HTI $=0.78, \mathrm{n}$ 
$249=9$ head turns, S1 Fig.). Concordantly, there was no significant group-level bias based on

250 individual HTIs, irrespective of sex and arousal (Table 1). Also, when considering all 50

251 stimulus presentations together, we found no significant group-level bias (mean $=0.091$; t-test: $t$

$252=1.228, \mathrm{p}=0.240)$.

253 On investigating possible methodological confounding factors, a binomial GLMM

254 analysis revealed that the direction of head turns was significantly influenced by Order (z-score $=$

$2552.14, \mathrm{p}=0.032$ ) and Initial Ear Position (position right: $\mathrm{z}$-score $=4.97, \mathrm{p}<0.001$, position

256 straight: $z$-score $=1.80, p=0.072)$, but not by Sex $(z$-score $=-0.585, p=0.559)$ and Arousal $(z-$

257 score $=0.692, \mathrm{p}=0.489)$. On calculating a further binomial GLMM, adding the interaction term

258 Order*Initial Ear Position, we found no significant interactions between these two factors (z-

259 score $<0.551, \mathrm{p} \geq 0.582$ ). Thus, we further analysed the two influencing factors separately.

260

\section{Habituation effect}

Non-linear regression analysis of the head turn response using the one-phase decay 263 function revealed a decline below $50 \%$ after the first 7 stimulus presentations which levelled off at around $32.45 \%$ (i.e. 5 responding subjects) without converging to zero (non-linear regression: $\mathrm{r}^{2}=0.453, \mathrm{Y0}=89 \%$, plateau $\left.=32.45 \%, \mathrm{~K}=0.167\right)$. In contrast, the distribution of the HTI across stimulus presentations showed an inverse progression (Fig. 3). Thus, the one decay

267 function increased for the first 7 stimulus presentations and reached its plateau at a HTI of 0.15 $268 \quad\left(\mathrm{r}^{2}=0.09, \mathrm{Y} 0=-0.77\right.$, plateau $\left.=0.15 \mathrm{~K}=0.243\right)$.

To further characterise the effect of habituation, we separately calculated the individual

270 HTIs for the first 7 presentations and the following 7 playback presentations (Fig. 4). The low

271 number of responses did not allow for a statistical analysis on an individual-level. The analysis 
272 for all 15 subjects revealed significant group-level biases to the left for the first 7 presentations

273 (negative HTIs; Wilcoxon test vs $0: \mathrm{W}=16, \mathrm{p}=0.038$ ) but no significant group-level bias for the

274 following 7 presentations (Wilcoxon test vs $0: \mathrm{W}=50.5, \mathrm{p}=0.097$ ). Thereby the HTIs

275 significantly shifted to the right when comparing the first with the following 7 presentations

276 (Wilcoxon test: $\mathrm{W}=10.5, \mathrm{p}=0.008$ )

277 Concordantly, when only considering the very first head turn response of each individual 278 and calculating HTIs at the group-level, we found more left than right head turn responses, with 279 negative HTIs for all, but one, subgroups. The biases were however moderate (median absolute 280 value: HTI $=-0.27)$ and did not differ statistically from chance (Table 2 ).

281

282 Ear position

283 We used a very conservative method to define ear position asymmetries; thereby every 284 visible deflection from a parallel ear position was noted (Fig. 2 indicating a small deviation from 285 parallel to the left). In total, there were only 45 cases in which a head turn response was preceded 286 by a parallel ear position, in 82 cases the right ear was closer to the loudspeaker and in 163 cases 287 the left ear was closer to the loudspeaker. In an average $64 \%$ of cases a head turn was preceded 288 by a same-side ear position; this was significantly higher than expected by chance (t-test vs $50 \%$ : $289 \mathrm{t}=3.741, \mathrm{p}=0.002$; Fig. 5). When calculating individual HTIs based only on the occasions 290 when the ears were parallel, the HTIs did not differ significantly from zero (Wilcoxon test vs 0 : $291 \mathrm{~W}=60.50, \mathrm{p}=0.281)$. 292

\section{Discussion}


295 in responses to repeated playback-presentations of kitten calls conveying either low or high

296 arousal. Only two cats (one male, one female) showed a significant turning bias in response to

297 low arousal kitten calls. Thus, we revealed neither consistent individual turning biases nor

298 laterality at group-level, irrespective of sex or arousal category. Only, when splitting the data set

299 based on habituation, could we reveal a head turn asymmetry at group-level. Overall, subjects

300 turned more to the left side during the first seven presentations, when responsiveness was still

301 high. Afterwards, the subjects turned more to the right side. To the best of our knowledge, we are

302 the first to report a shift in head turn asymmetries during habituation to repeated sound

303 presentations. However, findings have to be considered with caution as we also revealed a

304 significant influence of the ear position prior to responding, with about $64 \%$ of the head turn

305 responses being preceded by a same-side ear position (chance level: $50 \%$ ). The result of a

306 binomial GLMM showed that both factors did not interact with each other, thus, in the following,

307 we will discuss these two independent factors separately.

308

\section{Habituation effect}

310 The number of responding subjects significantly declined in the course of the experiments

311 and only within the first 7 presentations did more than $70 \%$ of subjects respond with a head turn,

312 the number subsequently declining to about $35 \%$, without converging to zero. When dividing our

313 dataset after the regression line dropped below 50\% responding subjects, we found opposite

314 turning biases at group-level. Whereas initially the head turn was to the left, which based on the

315 rationale of the head turn paradigm would indicate a higher involvement of the right hemisphere,

316 the HTIs based on the following 7 presentations were shifted to the right (not reaching a 
317 significant group-level bias). The initial right turning bias was confirmed when only considering

318 the very first response of each subject, the results were however not significant. The lack of

319 statistical power is based on the relatively small sample size, as the study-design was chosen to

320 assess individual biases, rather than to reveal asymmetries at the group-level based on only on

321 response per individual.

Although a habituation effect on the head turn asymmetry has not been reported so far,

conflicting results in domestic dogs might be interpreted in this way. Presenting only one call per

subject, Reinholz-Trojan and colleagues (2012) revealed left turning biases (indicating right

325 hemispheric dominance) towards dog barks. On the contrary, Sinicalchi and colleagues (2012;

326 2008), who assessed HTIs based on repeated $(\mathrm{n}=7)$ playback presentations, revealed right

327 turning biases towards dog barks. The authors excluded the subsequent playback presentations,

328 due to habituation to the playback stimuli, using a similar approach as in our study. The

329 discrepancy between the results of the two research groups may indicate a decreased dominance

330 of the right hemisphere and an increased involvement of the left hemisphere during habituation

331 to repeated presentations of species-specific calls in dogs. If so, the process would already

332 influence the head turn direction prior to an observable decline in responsiveness (i.e. percentage

333 of stimuli that induced a head turn response). Further research would be necessary to directly

334 link changes in arousal state of an individual to changes in head turn responses to repeated sound

335 presentations, such as monitoring the heart rate. One example of changes in turning biases due to

336 habituation has been reported in wombats (Descovich et al. 2013). Thereby, the habituation to

337 the experimental set-up (without playback presentation) influenced the turning behaviour of the

338 subjects, resulting in a change from left to right turning biases. The authors assumed that the

339 initial left turning biases (i.e. without playback presentations) might be related to vigilance 
340 behaviour (Descovich et al. 2013). Concordantly, Zimmer and Demmel (2000) reported in

341 humans that an initial right hemispheric activation in response to tone bursts diminishes due to

342 habituation to repeated presentations of the sounds. These findings were discussed to be based on

343 lateralised brain functions related to stress, emotions and motor preparation. This right

344 hemispheric dominance in processing rather negative emotions, such as fear, anxiety and

345 aggression has been found in several non-human vertebrate species (for review see Leliveld et al.

346 2013). Thus, in our study the initial high reactivity to the salient kitten calls (Aitkin et al. 1994;

347 Konerding et al. 2016), might have led to a right hemispheric lateralisation that subsequently

348 diminished during habituation to repeated sound presentations due to the decline in behavioural

349 reactivity based on changes in the emotional state.

350

351 Ear position

Small deflections of the ear position significantly affected head turn direction with more same-side head turns than expected by chance. As the cat has highly and quickly movable pinnae

354 (Populin \& Yin 1998), even an appropriate alignment of the body axis to the loudspeaker and the criteria that the ear position was parallel when the stimuli was played back did not guarantee that sound was perceived with similar loudness at both ears. The detailed frame-by-frame video analysis showed that within the reaction time of the experimenter the position of the pinnae slightly moved and only about $10 \%$ of head turn responses were confirmed to be preceded by a parallel orientation of the pinnae. When restricting the data set to these responses, we did not

360 find a head turn asymmetry. However, due to the small sample size, a splitting of the remaining

361 data set based on habituation was not feasible. Although same-side head turns occurred

362 significantly more often than expected by chance, same-side head turns did not occur in all cases. 
363 Thus, a strong lateralised behaviour should not have been completely covered by the influence of

364 the ear position. Still, this finding critically hampers the interpretation of the head turn paradigm

365 as indicator of auditory laterality in cats, as mere sound level differences between the ears may

366 have influenced turning responses (Heffner \& Heffner 1988).

367

368 General methodological considerations

The described influencing factors on the head turn response in cats, further strengthen the main criticism regarding the head turn paradigm (Fischer et al. 2009; Teufel et al. 2010; Teufel et al. 2007): It has not been revealed that in a given subject an asymmetry in head turn behaviour serves as a reliable indicator for neuronal processes related to lateralised perception of an auditory stimulus (Teufel et al. 2010). First, the neuronal origin of the head turn response has not been verified (Teufel et al. 2010). Although, species-specific vocalisations have been described to asymmetrically activate auditory cortex regions both in humans (Zatorre et al. 2002) and nonhuman primates (Poremba et al. 2004), the head turn response in cats may instead have a more subcortical origin (Beitel \& Kaas 1993; Teufel et al. 2010) and might be related to emotional processing rather than auditory perception per se (Basile et al. 2009b; Reinholz-Trojan et al. 2012; Siniscalchi et al. 2016; Xue et al. 2015). Mammalian infant cries, such as the kitten isolation call, have been described to activate specific subcortical regions, such as auditory

381 thalamic areas and the amygdala, which are commonly activated during emotional processing

382 (for review see Newman 2007). Furthermore, the initial direction of unconditioned head 383 orienting responses in cats has been found to be unaffected by bilateral ablation of the auditory 384 cortices (Thompson \& Masterton 1978). Instead it has been shown that the inferior colliculi of 385 cats play an important role in reflexive orientation to sound in space (e.g. Syka \& Straschill 
386 1970) which is based on inter-aural time and -level differences (Caird \& Klinke 1987). Second, it

387 has been postulated that specialised brain regions, rather than whole hemispheres, exhibit the

388 lateralisation that affects behavioural asymmetries (Wager et al. 2003). As brain regions might

389 be lateralised independently from one another (Wager et al. 2003), the inference from an

390 asymmetrical motor response to auditory lateralisation (at cortical level) is probably not as direct

391 as assumed by the rationale of the head turn paradigm. Our results support these considerations,

392 as both the arousal state (habituation) and subcortical processes (level differences between ears)

393 influence the head turn behaviour. These findings complicate the interpretation of the paradigm,

394 which generally assumes a turning response to the contralateral side of the dominant hemisphere.

\section{Conclusion}

We analysed for the first time potential auditory laterality in the domestic cat at 400 individual-level. Based on repeated sound presentations, domestic cats did not show a turning asymmetry to kitten calls either at individual- or group-level, irrespective of call category or sex of the listener. When splitting the data set based on an observed habituation effect, we revealed an initial left ear advantage. This finding is discussed to be related to a right hemispheric dominance during induced high behavioural reactivity. As additional confounding factor, we revealed the ear/ pinna position closer to the loudspeaker at sound onset. Due to the discussed

406 limitations of the head turn paradigm, we highlight the importance of a comparative,

407 neurological study to verify our results in the domestic cat. Our findings suggest that both the 
408 experimental sequence and the listening situation strongly influence the outcome of the head turn

409 paradigm and should be taken into account when evaluating auditory laterality at behavioural

410 level.

411

\section{Acknowledgements}

413 We wish to thank K. Möller for animal care and assistance during data collection, S. v.d.

414 Berg for technical support and B. Haßfurther for video analysis for inter-observer reliability as

415 well as F. Sherwood-Brock for professionally proofreading the English.

416

417 


\section{References}

419 Aitkin L, Tran L, and Syka J. 1994. The responses of neurons in subdivisions of the inferior $420 \quad$ colliculus of cats to tonal, noise and vocal stimuli. Experimental Brain Research 98:5364. $10.1007 / \mathrm{bf} 00229109$

422

423

424

425

426

427

428

429

430

431

432

433

434

435

436

437

438

Basile M, Boivin S, Boutin A, Blois-Heulin C, Hausberger M, and Lemasson A. 2009a. Socially dependent auditory laterality in domestic horses (Equus caballus). Animal Cognition 12:611-619. 10.1007/s10071-009-0220-5

Basile M, Lemasson A, and Blois-Heulin C. 2009b. Social and emotional values of sounds influence human (Homo sapiens) and non-human primate (Cercopithecus campbelli) auditory laterality. PLoS One 4:e6295. 10.1371/journal.pone.0006295

Beitel RE, and Kaas J. 1993. Effects of bilateral and unilateral ablation of auditory cortex in cats on the unconditioned head orienting response to acoustic stimuli. Journal of Neurophysiology 70:351-369.

Böye M, Güntürkün O, and Vauclair J. 2005. Right ear advantage for conspecific calls in adults and subadults, but not infants, California sea lions (Zalophus californianus): hemispheric specialization for communication? European Journal of Neuroscience 21:1727-1732. $10.1111 / \mathrm{j} .1460-9568.2005 .04005 . \mathrm{x}$

Caird D, and Klinke R. 1987. Processing of interaural time and intensity differences in the cat inferior colliculus. Experimental Brain Research 68:379-392.

Dehaene-Lambertz G, Dehaene S, and Hertz-Pannier L. 2002. Functional neuroimaging of speech perception in infants. Science 298:2013-2015. 
439 Descovich K, Reints Bok T, Lisle A, and Phillips C. 2013. Auditory laterality in a nocturnal, 440 fossorial marsupial (Lasiorhinus latifrons) in response to bilateral stimuli. Laterality: Asymmetries of Body, Brain and Cognition 18:32-43. 10.1080/1357650X.2011.626562

442 Eggermont JJ. 1998. Representation of spectral and temporal sound features in three cortical 443 fields of the cat. Similarities outweigh differences. Journal of Neurophysiology 80:27432764.

Fischer J, Teufel C, Drolet M, Patzelt A, Rubsamen R, von Cramon DY, and Schubotz R. 2009.

Ghazanfar AA, Smith-Rohrberg D, and Hauser MD. 2001. The role of temporal cues in rhesus monkey vocal recognition: orienting asymmetries to reversed calls. Brain, Behavior and Evolution 58:163-172. 10.1159/000047270

451

454

455

456

457

458

459

460

461 Orienting asymmetries and lateralized processing of sounds in humans. $B M C$ Neuroscience 10:14. 10.1186/1471-2202-10-14

\section{0lution 58:163-172. 10.1159/000047270}

Gil-da-Costa R, and Hauser MD. 2006. Vervet monkeys and humans show brain asymmetries for processing conspecific vocalizations, but with opposite patterns of laterality. Proceedings of the Royal SocietyB, Biological sciences 273:2313-2318. 10.1098/rspb.2006.3580

Hauser M, Agnetta B, and Perez C. 1998. Orienting asymmetries in rhesus monkeys: the effect of time-domain changes on acoustic perception. Animal Behaviour 56:41-47. 10.1006/anbe. 1998.0738

Hauser MD, and Andersson K. 1994. Left hemisphere dominance for processing vocalizations in adult, but not infant, rhesus monkeys: field experiments. Proc Natl Acad Sci USA 91:3946-3948.

Heffner RS, and Heffner HE. 1985. Hearing range of domestic cat. Hearing Research 19:85-88. $10.1016 / 0378-5955(85) 90100-5$ 
462 Heffner RS, and Heffner HE. 1988. Sound localization acuity in the cat: effect of azimuth, signal 463 duration, and test procedure. Hearing Research 36:221-232. 10.1016/03785955(88)90064-0

465

466

467

468

469

470

471

472

473

474

475

476

477

478

479

480

481

482

483

Hugdahl K, and Westerhausen R. 2015. Speech processing asymmetry revealed by dichotic listening and functional brain imaging. Neuropsychologia. 10.1016/j.neuropsychologia.2015.12.011

Konerding WS, Hedrich HJ, Bleich E, and Zimmermann E. 2012. Paw Preference Is Not Affected by Postural Demand in a Nonprimate Mammal (Felis silvestris catus). Journal of Comparative Psychology 126:15-22. 10.1037/a0024638

Konerding WS, Zimmermann E, Bleich E, Hedrich H-J, and Scheumann M. 2016. Female cats, but not males, adjust responsiveness to arousal in the voice of kittens. Bmc Evolutionary Biology 16:157. 10.1186/s12862-016-0718-9

Land R, Baumhoff P, Tillein J, Lomber SG, Hubka P, and Kral A. 2016. Cross-modal plasticity in higher-order auditory cortex of congenitally deaf cats does not limit auditory responsiveness to cochlear implants. Journal of Neuroscience 36:6175-6185. 10.1523/JNEUROSCI.0046-16.2016

Leliveld LM, Scheumann M, and Zimmermann E. 2010. Effects of caller characteristics on auditory laterality in an early primate (Microcebus murinus). PLoS One 5:e9031. 10.1371/journal.pone.0009031

Leliveld LMC, Langbein J, and Puppe B. 2013. The emergence of emotional lateralization: evidence in non-human vertebrates and implications for farm animals. Applied Animal Behaviour Science 145:1-14. 10.1016/j.applanim.2013.02.002 
484 Lemasson A, Koda H, Kato A, Oyakawa C, Blois-Heulin C, and Masataka N. 2010. Influence of 485 sound specificity and familiarity on Japanese macaques'(Macaca fuscata) auditory laterality. Behavioural Brain Research 208:286-289. 10.1016/j.bbr.2009.12.008

487

488

489

490

491

492

493

494

495

496

497

498

499

500

501

502

503

504

Ley RG, and Bryden M. 1982. A dissociation of right and left hemispheric effects for recognizing emotional tone and verbal content. Brain and Cognition 1:3-9. $10.1016 / 0278-2626(82) 90002-1$

Newman JD. 2007. Neural circuits underlying crying and cry responding in mammals. Behavioural brain research 182:155-165.

Ocklenburg S, Ströckens F, and Güntürkün O. 2013. Lateralisation of conspecific vocalisation in non-human vertebrates. Laterality: Asymmetries of Body, Brain and Cognition 18:1-31. 10.1080/1357650X.2011.626561

Palleroni A, and Hauser MD. 2003. Experience-dependent plasticity for auditory processing in a raptor. Science 299:1195. 10.1126/science.1078813

Populin LC, and Yin TC. 1998. Pinna movements of the cat during sound localization. Journal of Neuroscience 18:4233-4243.

Poremba A, Malloy M, Saunders RC, Carson RE, Herscovitch P, and Mishkin M. 2004. Speciesspecific calls evoke asymmetric activity in the monkey's temporal poles. Nature 427:448451.

Ratcliffe VF, and Reby D. 2014. Orienting asymmetries in dogs' responses to different communicatory components of human speech. Current Biology 24:2908-2912. 10.1016/j.cub.2014.10.030 
505 Reinholz-Trojan A, Włodarczyk E, Trojan M, Kulczyński A, and Stefańska J. 2012. Hemispheric 506 specialization in domestic dogs (Canis familiaris) for processing different types of acoustic stimuli. Behavioural Processes 91:202-205. 10.1016/j.beproc.2012.07.001

508 Romand R, and Ehret G. 1984. Development of sound production in normal, isolated and 509 deafened kittens during the first postnatal months. Developmental Psychobiology 17:629649. 10.1002/dev.420170606

511 Scheumann M, Roser A-E, Konerding W, Bleich E, Hedrich H-J, and Zimmermann E. 2012. Vocal correlates of sender-identity and arousal in the isolation calls of domestic kitten (Felis silvestris catus). Frontiers in Zoology 9:36. 10.1186/1742-9994-9-36

514 Scheumann M, and Zimmermann E. 2008. Sex-specific asymmetries in communication sound perception are not related to hand preference in an early primate. BMC Biol 6:3. $10.1186 / 1741-7007-6-3$

517 Siniscalchi M, Laddago S, and Quaranta A. 2016. Auditory lateralization of conspecific and 518 heterospecific vocalizations in cats. Laterality: Asymmetries of Body, Brain and Cognition 21:215-227. 10.1080/1357650X.2015.1116541

520 Siniscalchi M, Lusito R, Sasso R, and Quaranta A. 2012. Are temporal features crucial acoustic cues in dog vocal recognition? Animal Cognition 15:815-821. 10.1007/s10071-012-0506$\mathrm{X}$

523 Siniscalchi M, Quaranta A, and Rogers LJ. 2008. Hemispheric specialization in dogs for processing different acoustic stimuli. PLoS One 3:e3349. 10.1371/journal.pone.0003349

525 Syka J, and Straschill M. 1970. Activation of superior colliculus neurons and motor responses 526 after electrical stimulation of the inferior colliculus. Experimental neurology 28:384-392. 10.1016/0014-4886(70)90175-5 
528 Taglialatela JP. 2007. Functional and structural asymmetries for auditory perception and vocal

529 production in nonhuman primates. Special Topics in Primatology 5:120-145.

$530 \quad 10.1016 / \mathrm{S} 1936-8526(07) 05005-1$

531 Teufel C, Ghazanfar AA, and Fischer J. 2010. On the relationship between lateralized brain 532 function and orienting asymmetries. Behavioral Neuroscience 124:437-445. $10.1037 / \mathrm{a} 0022814$

534 Teufel C, Hammerschmidt K, and Fischer J. 2007. Lack of orienting asymmetries in Barbary macaques: implications for studies of lateralized auditory processing. Animal Behaviour 73:249-255. 10.1016/j.anbehav.2006.04.011

Wager TD, Phan KL, Liberzon I, and Taylor SF. 2003. Valence, gender, and lateralization of functional brain anatomy in emotion: a meta-analysis of findings from neuroimaging. Neuroimage 19:513-531. 10.1016/S1053-8119(03)00078-8

Thompson GC, and Masterton RB. 1978. Brain stem auditory pathways involved in reflexive head orientation to sound. Journal of Neurophysiology 41:1183-1202.

544 Wells DL, and Millsopp S. 2012. The Ontogenesis of Lateralized Behavior in the Domestic Cat, Felis silvestris catus. Journal of Comparative Psychology 126:23-30. 10.1037/a0026522

546 Xue F, Fang G, Yang P, Zhao E, Brauth SE, and Tang Y. 2015. The biological significance of 547 acoustic stimuli determines ear preference in the music frog. Journal of Experimental Biology 218:740-747. 10.1242/jeb.114694 
549 Young G, Bowman J, Methot C, Finlayson M, Quintal J, and Boissonneault P. 1983.

550 Hemispheric specialization development: What (inhibition) and how (parents). Manual 551 specialization and the developing brain 7:119-140.

552 Young G, and Gagnon M. 1990. Neonatal laterality, birth stress, familial sinistrality, and 553 left-brain inhibition. Developmental Neuropsychology 6:127-150. $554 \quad 10.1080 / 87565649009540455$

555 Zatorre RJ, Belin P, and Penhune VB. 2002. Structure and function of auditory cortex: music and 556 speech. Trends in Cognitive Sciences 6:37-46. 10.1016/S1364-6613(00)01816-7

557 Zimmer H, and Demmel R. 2000. Habituation and laterality of orienting processes as reflected 558 by slow negative waves. Biological Psychology 53:161-176. 10.1016/S0301$559 \quad 0511(00) 00048-X$

560 Zuur AF, Ieno EN, Walker NJ, Saveliev AA, and Smith GM. 2009. Zero-truncated and zero561 inflated models for count data. Mixed effects models and extensions in ecology with R:

562 Springer, 261-293.

563

564 
Figure 1

Representative low (A) and high (B) arousal kitten calls of the same sender

Depicted are oscillograms and spectrograms of each call from Konerding et al., 2016.
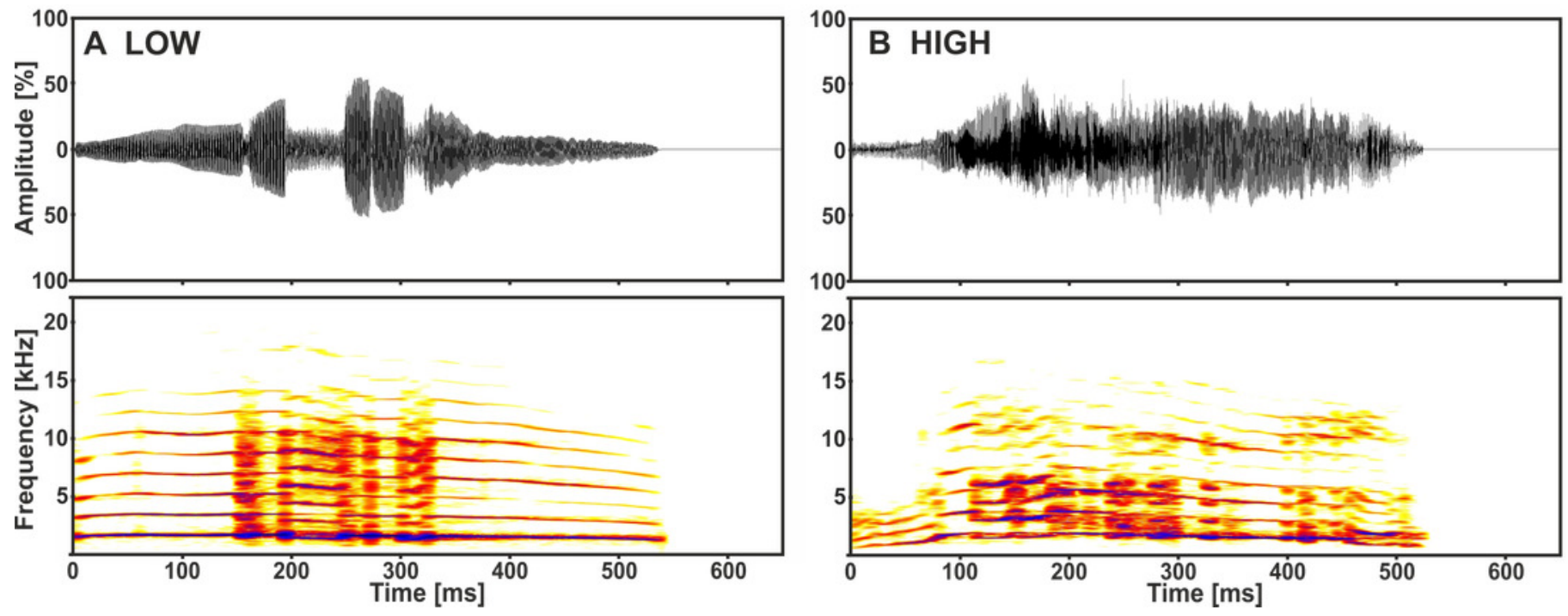


\section{Figure 2}

\section{Schematic drawing of the playback set-up}

Depicted is a cat inside the experimental cage, in front of a drinking bottle. The cage is equipped with two wire meshes, restricting the freedom of head movements. The cage is surrounded by sound attenuating foam and the loudspeaker is placed inside an opening $180^{\circ}$ behind the subject. The playback onset is indicated by the flashing of a diode (red). Cage bars were used as reference for parallel position of the tips of the pinnae. In this example pinnae was counted as a small deviation from parallel to the left).

*Note: Auto Gamma Correction was used for the image. This only affects the reviewing manuscript. See original source image if needed for review. 


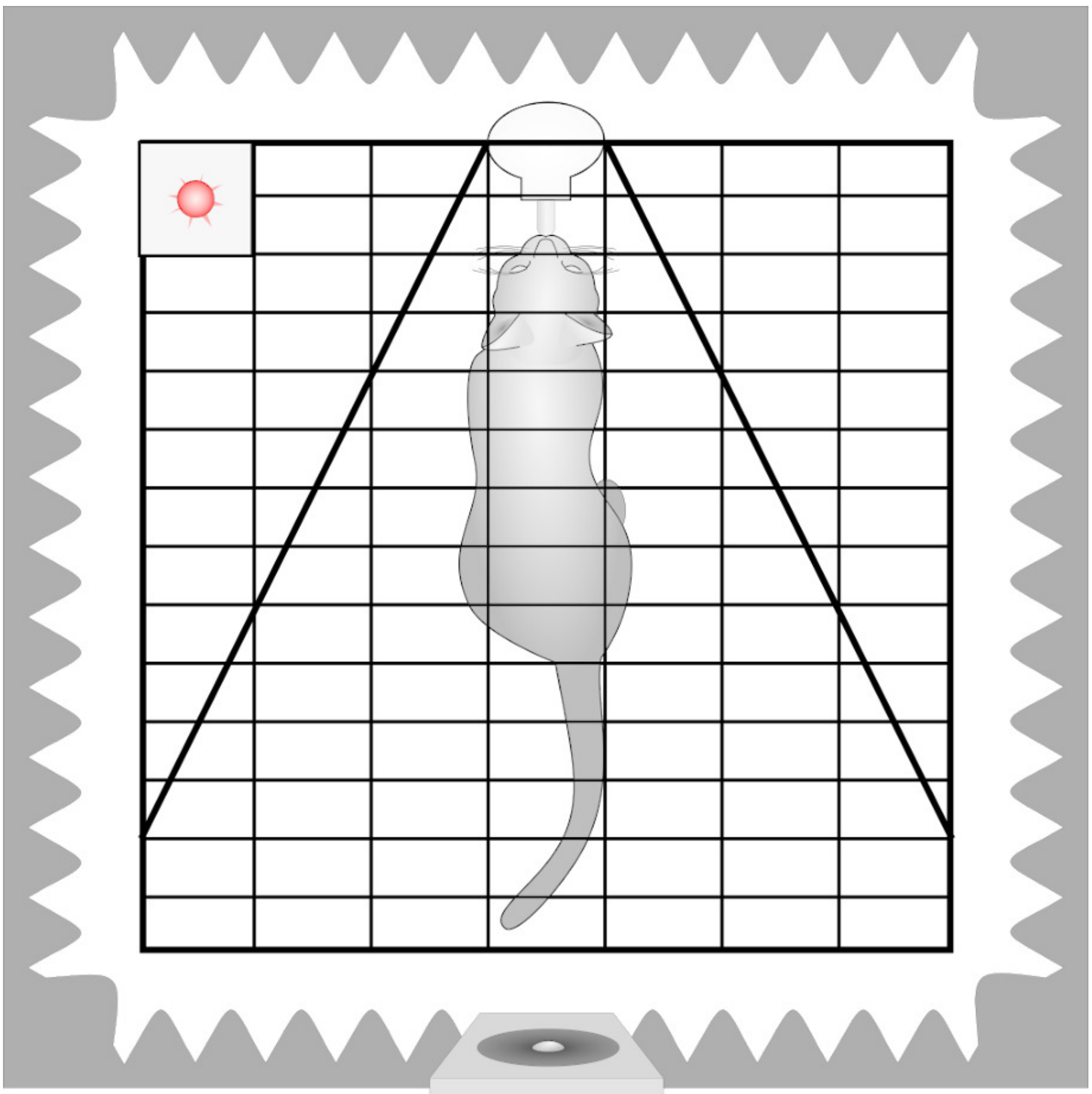


Figure 3

Influence of habituation on the number of responding subjects and the head turn index (HTI)

Given are the change of responding subjects (blue) and the HTI (red) during 50 playback presentations. The regression line (one-phase decay) indicates a habituation (50\% responding subjects) within the first 7 presentations (grey box) which is accompanied by a zero-crossing of the HTI-regression line.

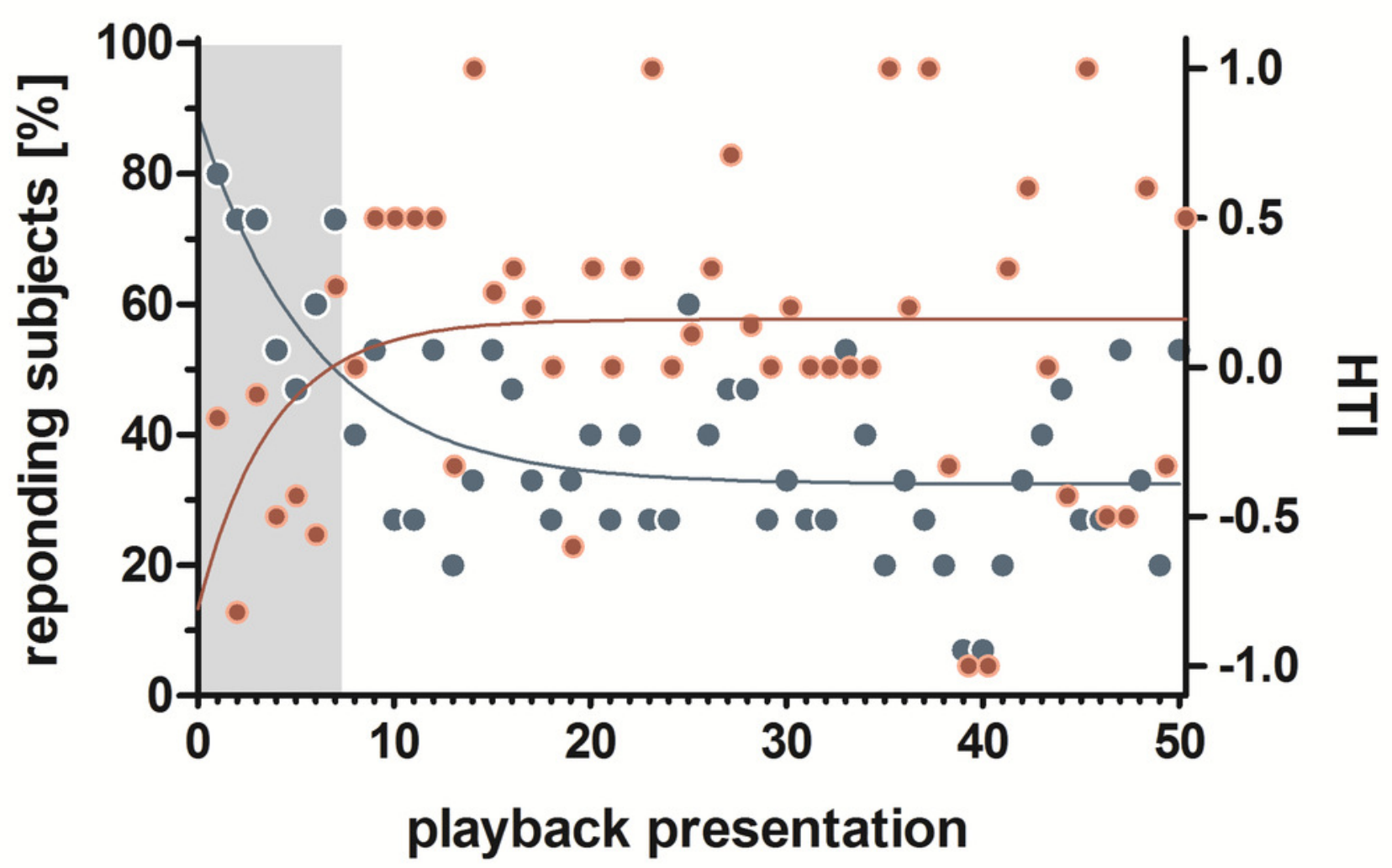


Figure 4

The head turn indices (HTI) based on the first 7, but not on the following 7, playback presentations shows a group-level bias to the left

Depicted are individual data points (circles) connected by lines and the respective group medians (stars). Medians were chosen, as the data of the "following 7" were not normally distributed. **Wilcoxon signed rank test: $p=0.008$; *Wilcoxon vs. $0: p=0.038$.

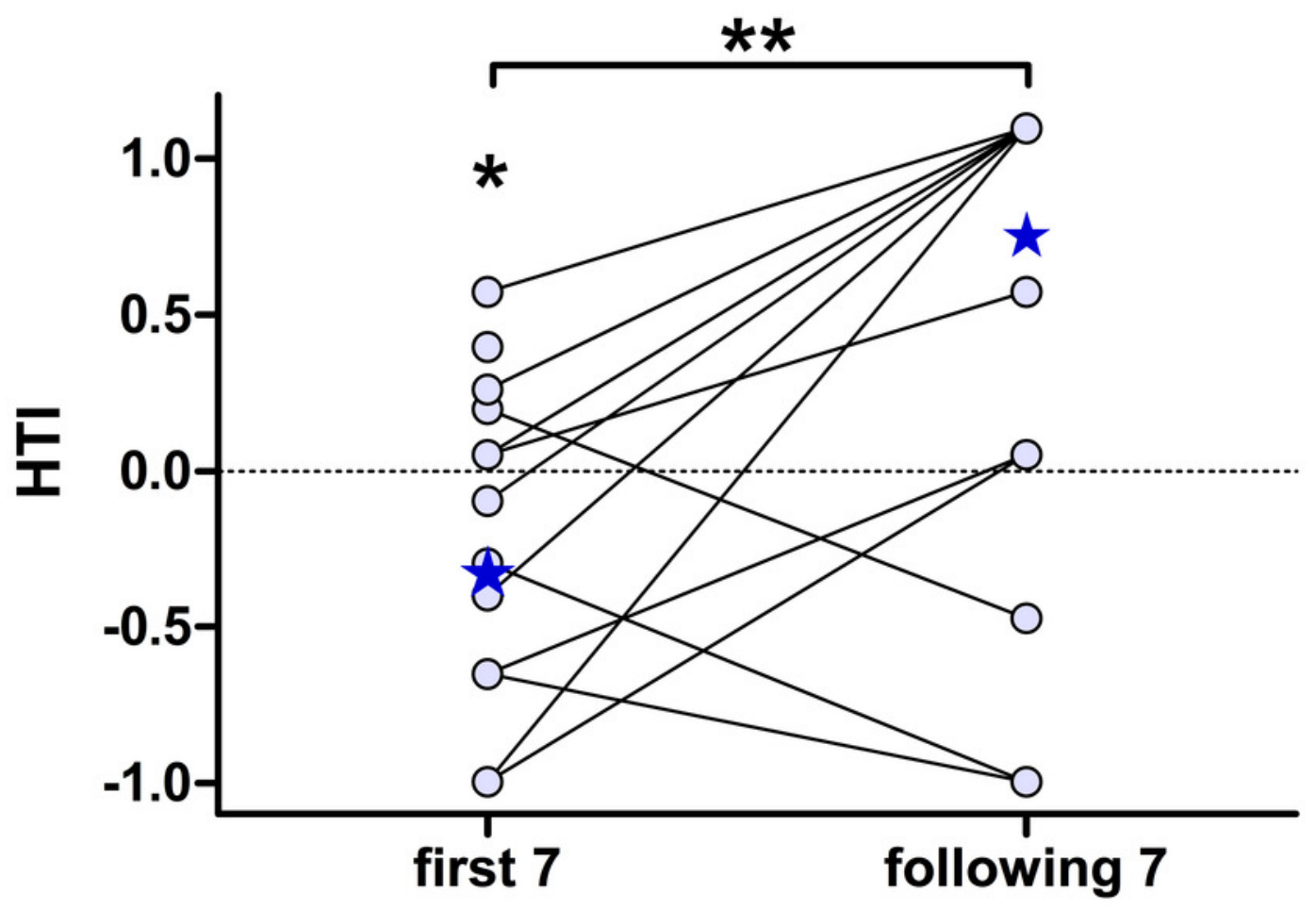


Figure 5

Influence of initial ear position on the direction of the head turn

Depicted are means $(\mathrm{N}=15)$ and standard deviations (whisker), with individual data points (circles). **onesample-t-test: $p=0.002$

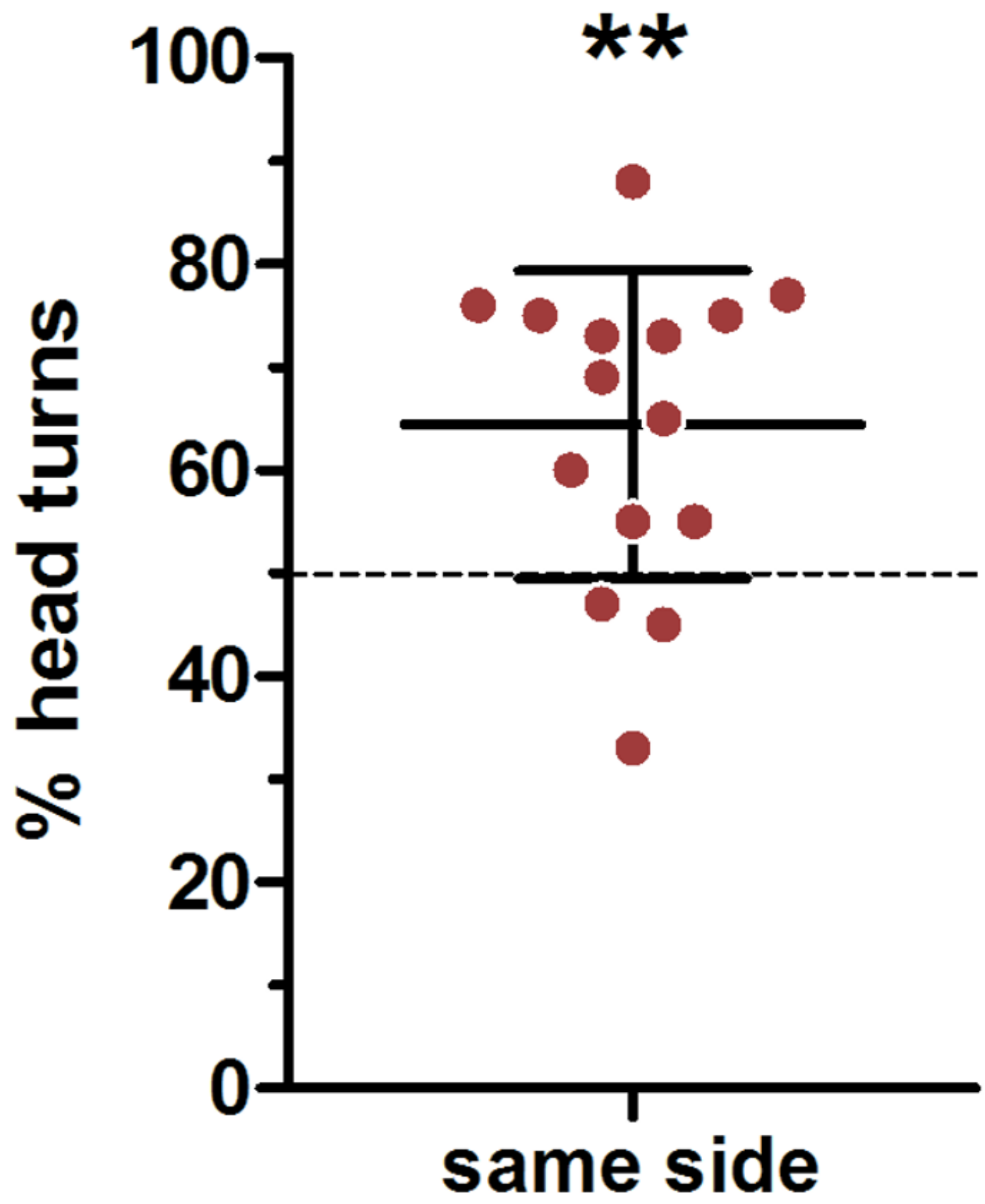




\section{Table $\mathbf{1}$ (on next page)}

Individual head turn indices did not differ significantly from chance at the group level 


\begin{tabular}{lllll}
\hline Sex & Arousal & Mean HTI & t-value & p-value \\
\hline \multirow{2}{*}{ Male } & low & 0.186 & 1.432 & 0.195 \\
& high & 0.139 & 2.252 & 0.059 \\
\multirow{2}{*}{ Female } & low & 0.114 & 0.643 & 0.544 \\
& high & -0.049 & -0.368 & 0.726 \\
\hline
\end{tabular}

2

3

4 
Table 2 (on next page)

First head turn response to low and high arousal kitten calls 


\begin{tabular}{llcccc}
\hline \multirow{4}{*}{ Male } & & left turn & right turn & HTI & p-value \\
\hline \multirow{4}{*}{ Female } & low & 3 & 5 & 0.25 & 0.727 \\
& high & 7 & 1 & -0.75 & 0.070 \\
& & 10 & 6 & -0.25 & 0.454 \\
& low & 5 & 2 & -0.43 & 0.453 \\
& high & 4 & 3 & -0.14 & 1.000 \\
both & low & 9 & 5 & -0.29 & 0.424 \\
& high & 8 & 7 & -0.07 & 1.000 \\
& & 11 & 4 & -0.47 & 0.118 \\
& & 19 & 11 & -0.27 & 0.200 \\
\hline
\end{tabular}

2

3 